\title{
Efecto antihipertensivo del extracto de Piper aduncum 'matico' sobre la hipertensión inducida por L-NAME en ratones
}

\author{
Piper aduncum ‘matico' extract antihypertensive effect on L-NAME- \\ induced hypertension in mice
}

\begin{abstract}
Jorge Arroyo ${ }^{1,2}$, Renán Hañari, ${ }^{3,5}$, Aldo Tinco ${ }^{3,4}$, Deyvis Baca ${ }^{3,5}$, Lester Domínguez ${ }^{2,5}$, Jesús Buendía

Instituto de Investigaciones Clínicas, Facultad de Medicina, Universidad Nacional Mayor de San Marcos, Lima, Perú.

${ }^{2}$ Laboratorio de Farmacología, Facultad de Medicina, Universidad Nacional Mayor de San Marcos, Lima, Perú. ${ }^{3}$ Laboratorio de Química Orgánica, Facultad de Farmacia y Bioquímica, Universidad Nacional Mayor de San Marcos, Lima, Perú. ${ }^{4}$ Laboratorio de Farmacologia, Escuela de Formación Profesional de Farmacia y Bioquímica, Universidad Nacional de San Cristóbal de Huamanga, Ayacucho, Perú.

${ }^{5}$ Unidad de Posgrado, Facultad de Farmacia y Bioquímica, Universidad Nacional Mayor de San Marcos, Lima, Perú.
\end{abstract}

\begin{abstract}
Resumen
Introducción: El Piper aduncum es una planta conocida como matico. Se le atribuye efectos antihipertensivo, antiinflamatorio, cicatrizante. Objetivos: Determinar el efecto antihipertensivo del extracto de Piper aduncum 'matico', sobre la hipertensión inducida por L-NAME, en ratones. Diseño: Experimental. Lugar: Facultades de Medicina y de Farmacia y Bioquímica, Universidad Nacional Mayor de San Marcos, Lima, Perú. Material biológico: Extracto etanólico de las hojas de Piper aduncum y ratas. Intervenciones: Se utilizó seis grupos de seis ratones Muss musculus cada uno, uno sin hipertensión (control negativo) y cuatro con hipertensión inducida por L-NAME: un control positivo y tres grupos para las dosis de 50, 150 y $300 \mathrm{mg} / \mathrm{kg}$, respectivamente. El tratamiento se realizó por vía oral, una vez por dia, durante 25 dias. Las mediciones de la presión arterial sistólica (PAS), presión arterial diastólica (PAD) y presión arterial media (PAM) fueron realizadas dos veces por semana (martes y viernes) y se consideró las mediciones entre los días 19 y 23 de iniciado el tratamiento. Principales medidas de resultados: Actividad antihipertensiva. Resultados: En los dias 19 y 23 se observó los mejores niveles de presión arterial en el grupo control y los experimentales, correspondiendo a las mediciones 6 y 7 . La eficacia antihipertensiva para enalapril fue 24,1 a $20,6 \%$, respectivamente, seguida por matico, entre 24,9 y $13,7 \%$ ( $p<0,05$ ). Conclusiones: En las condiciones experimentales, se demostró la actividad antihipertensiva del extracto etanólico de hojas de Piper aduncum 'matico'.

Palabras clave: Agentes antihipertensivos, matico, hipertensión.
\end{abstract}

\section{Abstract}

Introduction: Piper aduncum also known as matico is credited with antihypertensive, anti-inflammatory, healing effects. Objectives: To determine the antihypertensive activity of Piper aduncum 'matico' extract on L-NAME-induced hypertension in mice. Design: Experimental. Setting: Faculty of Medicine, and Faculty of Pharmacy and Biochemistry, National University of San Marcos, Lima, Peru. Biological material: Piper aduncum ethanolic extract leaves and mice. Interventions: Six groups of six Muss musculus mice each were used, one without hypertension (negative control) and four with L-NAME-induced hypertension: one positive control and three groups for 50,150 and $300 \mathrm{mg} / \mathrm{kg}$ doses, respectively. Treatment was given orally once daily for 25 days. Measurements of systolic blood pressure (SBP), diastolic blood pressure (DBP) and mean arterial pressure (MAP) were performed twice weekly (Tuesday and Friday) and measurements between 19 and 23 days of treatment were considered. Main outcome measures: Antihypertensive activity. Results: Higher blood pressure levels were observed at days 19 and 23 in the control and experimental groups that corresponded to measurements 6 and 7. The antihypertensive efficacy to enalapril was 24.1 to $20.6 \%$, followed by matico between 24.9 and $13.7 \%$ ( $p$ $<0.05)$. Conclusions: Antihypertensive activity of Piper aduncum 'matico' extract was demonstrated under experimental conditions.

Key words: Antihipetensive agents, matico, hypertension.

An Fac med. 2012;73(4):275-80 


\section{INTRODUCCIÓN}

La hipertensión arterial (HTA) es uno de los principales problemas de salud pública debido a su alta prevalencia y asociación con un incrementado riesgo de morbimortalidad por causas cardiovasculares. Se estima que en el año 2000 había 972 millones de hipertensos en el mundo ${ }^{(1)}$. La hipertensión arterial sistémica (HAS) es de las enfermedades crónicas de mayor prevalencia en México, ya que afecta al 30,8\% de la población de entre 20 a 69 años de edad $^{(2)}$. En el Perú, las ciudades de la costa presentan mayores prevalencias $(27,3 \%)$ que las de la sierra $(18,8 \%)$ y selva $(22,1 \%)$, en población adulta ${ }^{(3)}$.

El Piper angustifolium R. EF P. o Piper aduncum, o Piper elongatum, es una planta conocida como matico ${ }^{(4)}$, cordoncillo, moho-moho, hierba do soldado ${ }^{(5)}$. Sus hojas y ramas contienen aceites esenciales, ácido artánico, resinas, sustancias amargas (maticina), taninos, alcaloides, saponinas, flavonoides triterpenoides ${ }^{(6)}$ (tabla 1). Los taninos poseen una acción inhibitoria de tipo no competitivo de la ECA ${ }^{(7)}$; los flavonoides tienen propiedades antioxidantes y protectoras de la membrana celular ${ }^{(8)}$ y disminuyen la HTA por la inhibición de la fosfodiesterasa, que destruye el AMPc mediante la apertura de su anillo. El resultado de esta inhibición es un aumento en el flujo de agua de la sangre en la célula tubular, de la que puede escapar a la orina. La eliminación de agua reduce la presión arterial. Los flavonoides son compuestos muy eficientes para este propósito, ya que inhiben también la enzima clave en la síntesis de prostaglandina (PG) por la ciclooxigenasa $(\mathrm{COX})^{(9)}$.

Se ha demostrado que las saponinas contribuyen a disminuir las complicaciones de la HTA, al aumentar el gasto cardíaco, recuperación de la función diastólica y protección de los cardiomiocitos frente al daño hipóxico; también, por reducción la sobrecarga de Ca2 + intracelular ${ }^{(10)}$; las saponinas pudieran derivarse de la recolección de residuos con el estrés oxidativo provocado por la generación y acumulación de especies reactivas de oxígeno (ROS), el alivio de la lesión por isquemia de miocardio y muerte de las células cardíacas ${ }^{(11)}$. Se ha encontrado que los productos alimenticios ricos en compuestos fenólicos poseen propiedades farmacológicas hipotensoras y antioxidantes ${ }^{(12)}$. Existen fármacos estándares para tratamientos farmacológicos, con limitación de costos y ciertos efectos adversos ${ }^{(13)}$, siendo necesario investigaciones para incorporar productos naturales alternativos para este fin. Esta investigación se realizó con el objetivo de determinar la eficacia antihipertensiva de los extractos de Piper aduncum 'matico', en ratones con inducción de hipertensión por L-NAME.

\section{MÉTODOS}

Las hojas de Piper aduncum 'matico' fueron colectadas de las ciudad de La Merced, departamento de Junín, y llevadas a sequedad a una temperatura menor de $40{ }^{\circ} \mathrm{C}$; se las pulverizó en molino, luego se maceró en alcohol etílico de $96^{\circ}$, durante 8 días; con agitación constante todos los días, se filtró y evaporó el solvente a temperatura de $\leq 40^{\circ} \mathrm{C}$, hasta obtener un residuo untuoso con peso constante, al cual se le denominó extracto etanólico. La detección de metabolitos secundarios se realizó mediante pruebas químicas de caracterización (reacción de Dragendorf, reacción de Shinoda, prueba de la espuma, reacción del tricloruro férrico, gelatina, Lieberman-Burchard, ninhidrina y Molish), según Lock ${ }^{(14)}$ (tabla 1).

Se utilizó ratones albinos machos cepa Muss musculus, con peso promedio de $35 \pm 5 \mathrm{~g}$, procedentes del Instituto Nacional de Salud (Lima, Perú), y que fueron mantenidos en el Bioterio de la Facultad de Medicina de la Universidad Nacional Mayor de San Marcos, con condicionamiento previo de 48 horas, con agua y alimento ad líbitum, ciclo luz-día de 12 horas y temperatura de 22 a $26^{\circ} \mathrm{C}$.

Se usó el modelo de hipertensión por L-NAME, teniendo en cuenta lo realizado por Sánchez-Mendoza y $\mathrm{col}^{(15)}$, con modificación del animal experimental y el sensor para ratón, que es usado ampliamente para estudiar la fisiopatología de la hipertensión arterial ${ }^{(16)}$.

Se utilizó 36 ratones machos distribuidos según el siguiente diseño experimental: 1) normal solvente $10 \mathrm{~mL} / \mathrm{kg}$; 2) L-NAME $50 \mathrm{mg} / \mathrm{kg}$ (LN) vía oral durante 5 días; 3) $\mathrm{LN}+$ enalapril $20 \mathrm{mg} /$ $\mathrm{kg}$; 4) $\mathrm{LN}+$ matico $50 \mathrm{mg} / \mathrm{kg}$; 5) $\mathrm{LN}+$ matico $150 \mathrm{mg} / \mathrm{kg}$; 6) $\mathrm{LN}+$ matico 300 $\mathrm{mg} / \mathrm{kg}$. Los animales previamente fueron adaptados a pernoctar en el cepo,

Tabla 1. Ensayo fitoquímico del extracto etanólico de las hojas de Piper aduncum 'matico'.

\begin{tabular}{ccc} 
Reacciones & Metabolito secundario & Matico \\
Dragendorff & Alcaloides & ++ \\
Mayer & Alcaloides & ++ \\
Shinoda & Flavonoides & +++ \\
Índice de espuma & Saponinas & ++ \\
Cloruro férrico & Compuestos fenólicos & ++ \\
Olor & Aceites & ++ \\
Gelatina & Taninos & ++ \\
Liebermann & Esteroides & + \\
Ninhidrina & Aminoácidos & - \\
Bortranger & Ouinonas & + \\
\hline
\end{tabular}

$(-)=$ Ausencia,$(+)=$ Poca cantidad.

$(++)=$ Regular cantidad,$(+++)=$ Bastante cantidad. 
Tabla 2. Valores medios \pm error estándar de valores de presión arterial y porcentaje de eficacia antihipertensiva en ratones.

\begin{tabular}{|c|c|c|c|c|c|c|c|}
\hline M & Tratamiento & PS & \%EAPS & PD & $\%$ EAPD & PM & \%EAPM \\
\hline 1 & Normal & $108,4 \pm 1,1$ & 0,0 & $87,0 \pm 1,1$ & 0,0 & $94,1 \pm 1,3$ & 0,0 \\
\hline 1 & L-NAME (LN) & $158,4 \pm 1,1$ & 0,0 & $117,0 \pm 1,1$ & 0,0 & $130,8 \pm 1,3$ & 0,0 \\
\hline 1 & LN + enalapril 20 mg/kg & $120,2 \pm 1,6$ & 24,1 & $115,1 \pm 1,5$ & 1,6 & $116,8 \pm 1,8$ & 10,7 \\
\hline 1 & $\mathrm{LN}$ + matico $50 \mathrm{mg} / \mathrm{kg}$ & $122,3 \pm 5,1$ & 22,8 & $122,9 \pm 3,4$ & $-5,1$ & $122,7 \pm 4,3$ & 6,2 \\
\hline 1 & $\mathrm{LN}+$ matico 150 mg/kg & $120,1 \pm 0,6$ & 24,2 & $96,0 \pm 0,6$ & 17,9 & $104,0 \pm 0,3$ & 20,4 \\
\hline 1 & $\mathrm{LN}+$ matico 300 mg/kg & $118,9 \pm 4,0$ & 24,9 & $109,2 \pm 2,8$ & 6,7 & $112,4 \pm 6,8$ & 14,0 \\
\hline 2 & Normal & $106,2 \pm 0,9$ & 0,0 & $86,6 \pm 0,9$ & 0,0 & $93,1 \pm 3,3$ & 0,0 \\
\hline 2 & L-NAME (LN) & $146,2 \pm 0,9$ & 0,0 & $119,6 \pm 0,9$ & 0,0 & $128,4 \pm 3,3$ & 0,0 \\
\hline 2 & $\mathrm{LN}+$ enalapril 20 mg/kg & $116,0 \pm 1,7$ & 20,6 & $109,8 \pm 1,7$ & 8,1 & $111,9 \pm 2,1$ & 12,9 \\
\hline 2 & $\mathrm{LN}$ + matico 50 mg/kg & $121,0 \pm 4,7$ & 17,2 & $117,8 \pm 4,7$ & 1,4 & $118,9 \pm 7,2$ & 7,4 \\
\hline 2 & $\mathrm{LN}+$ matico 150 mg/kg & $126,2 \pm 1,7$ & 13,7 & $116,7 \pm 1,7$ & 2,4 & $119,8 \pm 8,8$ & 6,7 \\
\hline 2 & $\mathrm{LN}+$ matico 300 mg/kg & $120,8 \pm 4,1$ & 17,4 & $103,1 \pm 4,7$ & 13,8 & $109,0 \pm 2,4$ & 15,2 \\
\hline 3 & Normal & $103,1 \pm 2,4$ & 0,0 & $89,3 \pm 8,3$ & 0,0 & $93,9 \pm 9,0$ & 0,0 \\
\hline 3 & L-NAME (LN) & $123,6 \pm 5,1$ & 0,0 & $100,0 \pm 3,4$ & 0,0 & $107,8 \pm 4,1$ & 0,0 \\
\hline 3 & $\mathrm{LN}+$ enalapril 20 mg/kg & $113,8 \pm 5,1$ & 7,9 & $95,7 \pm 2,1$ & 4,3 & $101,7 \pm 2,9$ & 5,6 \\
\hline 3 & $\mathrm{LN}$ + matico 50 mg/kg & $117,3 \pm 5,7$ & 5,0 & $95,9 \pm 3,6$ & 4,1 & $103,0 \pm 4,2$ & 4,5 \\
\hline 3 & $\mathrm{LN}+$ matico 150 mg/kg & $112,2 \pm 8,0$ & 9,2 & $103,2 \pm 7,6$ & $-3,3$ & $106,2 \pm 8,1$ & 1,5 \\
\hline 3 & $\mathrm{LN}+$ matico 300 mg/kg & $114,7 \pm 2,0$ & 7,2 & $94,0 \pm 1,6$ & 5,9 & $100,9 \pm 1,7$ & 6,4 \\
\hline
\end{tabular}

Donde \%EA = ((L-NAME - Tratamiento)/ L -NAME)*100). M = medición (1, 2 y 3 corresponden a la sexta (día 19), séptima (día 23) y octava (día 26) mediciones realizadas en el periodo de estudio); PS = presión sistólica; $\mathrm{PD}=$ presión diastólica; $\mathrm{PM}=$ presión media; \%EA = porcentaje de eficacia antihipertensiva. $\mathrm{p}<0,05$.

por lo menos 15 minutos dos veces al día, durante una semana, a temperatura ambiental; y a pernoctar en el cepo por lo menos 15 minutos diariamente durante otra semana, a temperatura de 30-35 ${ }^{\circ} \mathrm{C}$, porque el calor induce la vasodilatación de las venas de la cola del animal, permitiendo un mejor registro de presión arterial.

Se utilizó un equipo de medición de presión arterial incruenta marca LE 5002 Panlab, que contiene un microprocesador o sensor para captar indirectamente valores de presión arterial en la cola de ratón (sensor específico para ratones, porque también existe para ratas), y se registra valores de presión arterial sistólica (PAS), presión arterial diastólica (PAD), presión arterial media (PAM) expresados en $\mathrm{mmHg}$ y frecuencia cardíaca (FC) ${ }^{(17)}$. Se mantuvo un ambiente libre de ruidos para reducir el estrés en los animales, los que fueron tratados y colocados en el cepo para ratón sin traumatismos. Las mediciones de la presión arterial fueron realizadas dos veces por semana (martes y viernes) por las mañanas, en el mismo horario; el valor considerado fue el promedio de cinco lecturas consecutivas. Para la evaluación del efecto antihipertensivo, se utilizó los valores de los días 19, 23 y 26 de iniciado el tratamiento (coincidiendo con las mediciones 6, 7 y 8). Los datos de presión arterial $(\mathrm{mmHg})$ fueron sometidos a un análisis de varianza y la prueba de múltiples comparaciones de Duncan; para establecer la diferencia de medías por efecto del tratamiento, se hizo con ayuda del programa estadístico SPSS 13.0, año 2006; los hallazgos fueron considerados significativos a un valor $\mathrm{p}<0,05$.

La manipulación de los animales se hizo con los cuidados respectivos, a fin de evitar estrés, dolor, entre otros; y al final del experimento se les aplicó eu- tanasia, habiéndose utilizado la administración de pentobarbital $100 \mathrm{mg} / \mathrm{kg}$ vía intraperitoneal, por considerarse un método aceptable para roedores por el Report of the AVMA Panel on Euthanasia (1993) y el Euthanasia of Experimental Animals, de la Unión Europea ${ }^{(18)}$.

\section{RESULTADOS}

Los hallazgos del estudio fitoquímico preliminar mostraron una gran cantidad de compuestos fenólicos, alcaloides y flavonoides caracterizados mediante las pruebas de Dragendorff, Mayer, tricloruro férrico y de Shinoda, respectivamente, en el extracto atomizado del matico (tabla 1).

Las concentraciones del extracto de Piper aduncum 'matico' presentaron una reducción de la PAS, PAD y PAM en comparación con la del grupo control (figuras 1,2 y 3 ). 


\section{DISCUSIÓN}

La presión arterial sistémica alta es una enfermedad multifactorial, en la que la alteración del sistema renina angiotensina aldosterona (RAAS) tiene un papel central en el control de este trastorno. Por lo tanto, los fármacos que actúan como antagonistas o inhibidores de los diferentes mecanismos relacionados con el sistema RAAS son eficaces para el tratamiento de la hipertensión ${ }^{(19)}$.

En el presente estudio, el extracto de Piper aduncum reveló contener flavonoides, seguido por alcaloides, saponinas, taninos, aceites esenciales y esteroides (tabla 1); ha demostrado un efecto antihipertensivo relativamente efectivo con el extracto etanólico de matico, habiéndose realizado mediciones hasta la sexta semana de inducción de hipertensión con L-NAME, pero solamente son expresadas las medidas 6, 7 y 8 (correspondiente a los días 19, 23 y 26), observándose mayor reducción al usar las dosis de 50 y $150 \mathrm{mg} / \mathrm{kg}$ de extracto de matico.

La presión arterial presenta una regulación multifactorial, participando factores autocrinos, como el óxido nítrico $(\mathrm{ON})$, que regula procesos hipertensivos en modelos animales; el L-NAME hace evidente el efecto hipertensor (tabla 1), al inhibir la presencia de $\mathrm{ON}$; el tiempo de medición aproximado expuesto para este estudio es cercano al comunicado por Ramírez y col, quienes aluden reducción de los niveles de presión arterial y del $\mathrm{ON}$ en ratas a partir del día $15^{(16)}$. Por los resultados, se observa que los ratones con inducción de hipertensión posiblemente mostraron una sensibilidad aumentada del control barorreceptor arterial de la frecuencia cardiaca por incrementos agudos de la presión arterial y una atenuación significativa del reflejo de Bezold-Jarisch y el quimiorreflejo en los ratones, como lo explican Peotta y col, 2001, al evaluar los reflejos neurales a nivel cardiovascular ${ }^{(17)}$. La inhibición crónica de NO por L-NAME estimula la producción de tromboxano, al acti-

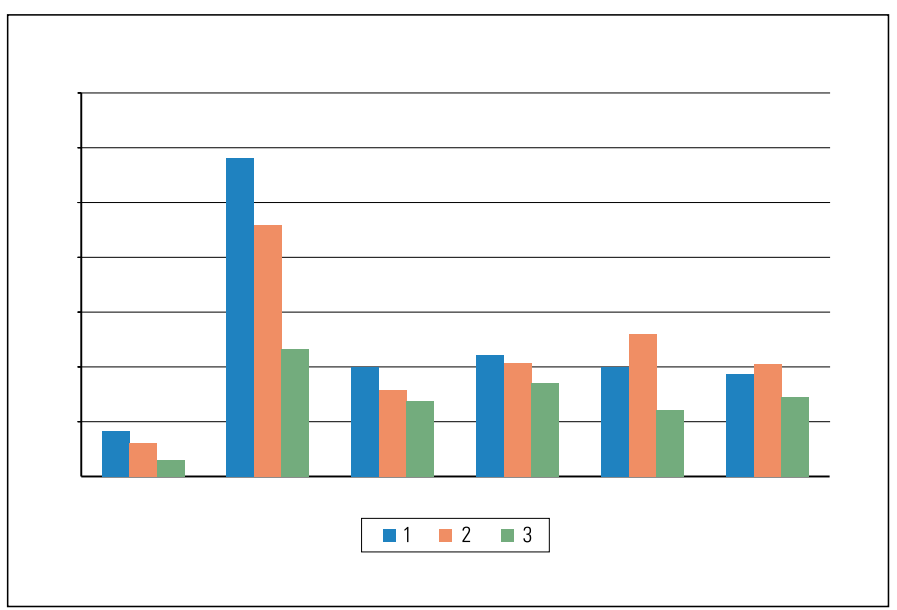

Figura 1. Nivel de presión arterial sistólica por efecto de tratamiento en ratones, donde 1, 2 y 3 corresponden a la sexta (día 19), séptima (día 23) y octava (día

26) mediciones de presión arterial realizadas en el periodo de estudio.

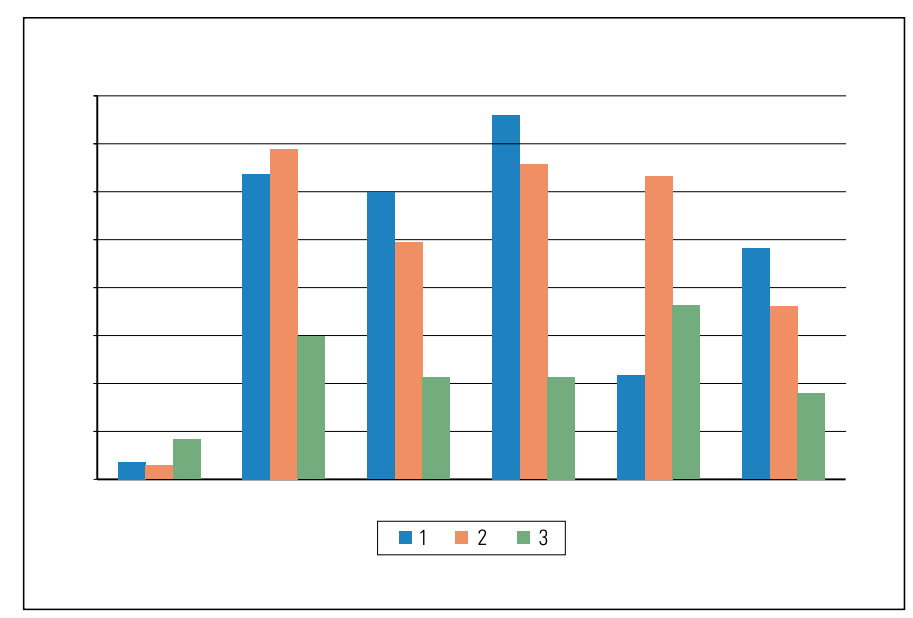

Figura 2. Nivel de presión arterial diastólica por efecto de tratamiento en ratones, donde 1,2 y 3 corresponden a la sexta (día 19), séptima (día 23) y octava (día

26) mediciones de presión arterial realizadas en el periodo de estudio.

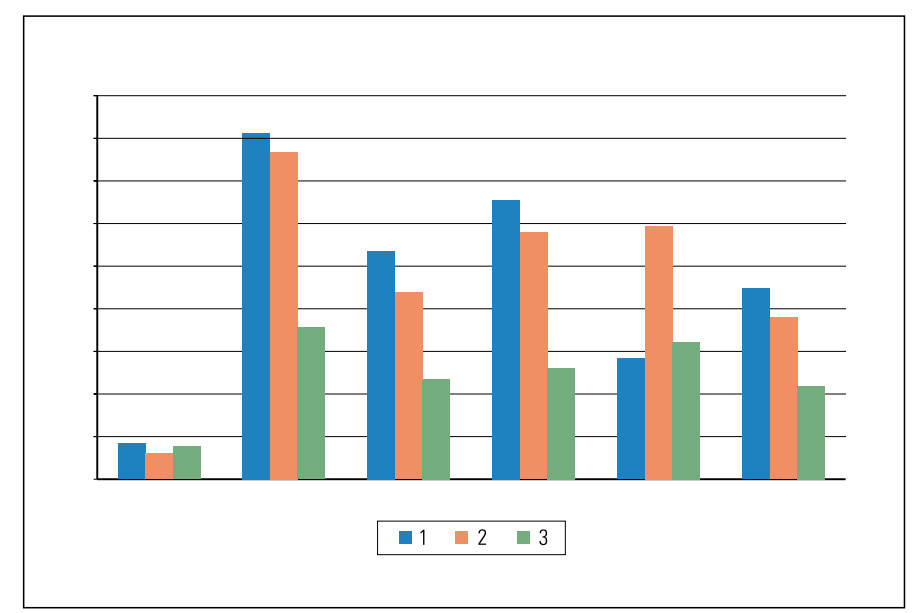

Figura 3. Nivel de presión arterial media por efecto de tratamiento en ratones, donde 1,2 y 3 corresponden a la sexta (día 19), séptima (día 23) y octava (día 26) mediciones de presión arterial realizadas en el periodo de estudio. 
varse los receptores de tromboxanosprostanoides (TP), lo que contribuye a la severidad de la hipertensión e hipertrofia cardíaca ${ }^{(18)}$. Se ha descrito que la administración de L-NAME produce un aumento de 20 a 40\% de la tensión arterial sistólica y diastólica en ratas. Además, el L-NAME ocasiona fibrosis cardíaca y nefropatía, características de daño de órgano blanco similares a la hipertensión humana. La hipertensión por L-NAME se debe sobre todo a la vasoconstricción, pues disminuye la síntesis de óxido nítrico y al aumento en la síntesis de renina ${ }^{(16)}$.

Por otro lado, los receptores TP atenúan el desarrollo de la hipertrofia renal y lesiones patológicas asociadas con la administración de L-NAME. En otros modelos de hipertensión, estudios previos demostraron contribuciones clave de la inhibición de los receptores TP sobre la gravedad de la elevación de la presión arterial, así como la hipertrofia cardíaca y fibrosis. En conjunto, estos datos sugieren que la inhibición del receptor TP es parte de una vía final común que conduce a un aumento de la presión arterial y de las lesiones de órganos diana de las enfermedades hipertensivas, con independencia de su causa principal ${ }^{(18)}$.

Es así que el efecto reductor de los niveles elevados de la presión arterial posiblemente se explicaría por el alto contenido de flavonoides, como se evidencia en la tabla 1; estos compuestos inducen relajación de los vasos sanguíneos al favorecer la presencia de algunas moléculas vasodilatadoras, como la acetilcolina, adenosin trifosfato (ATP) y adenosin difosfato (ADP), la sustancia $\mathrm{P}$, bradiquinina, histamina, trombina, serotonina y otras ${ }^{(19)}$. Asimismo, porque los flavonoides han demostrado inhibición de la proteinoquinasa C (PKC), inhibición de otras quinasas -como la fosfodiesterasa (PDE)-, y el bloqueo de la entrada de calcio ${ }^{(20)}$; también, por la inhibición de la enzima convertidora de angiotensina (IECA), inhibición de la síntesis de prostanoides e inducción de la relajación mediada por bradiquininas ${ }^{(21,22)}$, inhibición de la secreción de endotelina 1 (ET-1), potentes vasoconstrictores mucho más que la angiotensina II ${ }^{233}$. Además, siendo el óxido nítrico el mediador de la relajación dependiente del endotelio ${ }^{(24)}$, los flavonoides son capaces de incrementar la actividad del promotor de la sintasa del óxido nítrico endotelial (eNOS), así como el mensajero ARN (RNAm) de esta enzima, incrementando así la producción de óxido nítrico en cultivos celulares de células endoteliales humanas ${ }^{(24-26)}$.

En el estudio de Xu y col (27), ellos expresan que la cianidina-3-glicósido (un pigmento de las antocianinas o flavonoides fenólicos) induce la expresión de la sintasa de óxido nítrico endotelial y un aumento de la liberación de óxido nítrico de manera tiempo y dosis dependiente. Estos efectos mejoran la disfunción endotelial, normalizan la presión arterial y previenen la ateroesclerosis a largo plazo. A la procianida (miembro del grupo de las antocianinas) se le atribuye efectos antiinflamatorios y vasodilatadores ${ }^{(28)}$. Alarcón J, $2011^{(29)}$, al investigar el efecto hipotensor del extracto etanólico de Hibiscus sabdariffa Linn (Malvaceae), planta con contenidos altos de flavonoides tipo antocianinas, encontró gran efecto antihipertensivo, probablemente debido al antagonismo de calcio mediante la modulación de la activación de la vía de señalización derivado de óxido nítrico del endotelio / cGMP y enzima convertidora de angiotensina (ECA), a sus efectos diuréticos y natriuréticos, inhibición de la ECA, lo que evitaría la remodelación vascular cardíaca o renal. Se ha evidenciado que algunos flavonoides, aun cuando carecen de radicales hidroxilo, son capaces de inhibir la ECA ${ }^{(23)}$ y es posible que los flavonoides presentes en el extracto etanólico de Piper aduncum podrían ejercer dicho efecto inhibidor de la ECA, lo que hubiera contribuido en la disminución de las cifras de presión arterial.

La presencia de alcaloides en el extracto etanólico de matico sugiere su capacidad de activar los receptores colinérgicos muscarínicos, lo cual conlleva a un incremento del calcio intracelular de las células endoteliales, favoreciendo la síntesis de $\mathrm{NO}^{(30)}$. Otro de los componentes activos importantes del extracto etanólico de las hojas de Piper aduncum 'matico' son los taninos (polifenoles naturales), los cuales poseen una acción inhibitoria sobre la ECA ${ }^{(31)}$.

El modelo experimental con ratones se torna interesante al haber empleado el sensor especial en la cola del ratón a fin de obtener la variación de los niveles de presión arterial. Se ha logrado determinar el efecto hipotensor, debido probablemente a la presencia de elementos fitoquímicos presentes en el extracto etanólico de matico, como alcaloides, compuestos fenólicos, flavonoides y taninos; sin embargo, es necesario realizar futuros estudios que permitan conocer qué compuestos exactamente son los responsables. El consumo tradicional de estos productos puede ser beneficioso para la salud de la población, pero a la vez podría resultar en eventos negativos, por lo que se recomienda ampliar los estudios farmacológicos y toxicológicos.

En conclusión, se ha demostrado efecto antihipertensivo al administrar por vía oral el extracto etanólico de Piper aduncum 'matico' en ratones con inducción de hipertensión por L-NAME.

\section{REFERENCIAS BIBLIOGRÁFICAS}

1. Kearney $\mathrm{P}$, Whelton M, Reynolds $\mathrm{K}$, Muntner $\mathrm{P}$ Whelton P, He J. Global burden of hypertension: analysis of worlwide data. Lancet. 2005;365:21723.

2. Hernández M, Lezama M, Armando J, Mancha C, Barriguete-Meléndez J, Ortiz GR, et al. Guía de tratamiento farmacológico y control de la hipertensión arterial sistémica. Rev Mex Cardiol. 2011;22(Supl. 1):1A-21A.

3. Agusti R. Epidemiología de la hipertensión arterial en el Perú. Acta Med Peru. 2006;23(2):69-75.

4. MacBride JF. Flora del Perú. Ed. Publ. Field Museum Natural History Bot. Tomo II, 1937.

5. Soukup SJ. Vocabulario de los nombres vulgares de la flora peruana. Lima, Colegio Salesiano, 1970:70-2

6. Palacios VJ. Plantas medicinales nativas del Perú Lima, Concytec, 1993:22-5.

7. Ju-Chi L, Feng-Lin $\mathrm{H}$, Jen-Chen $\mathrm{T}$, Chana $\mathrm{P}, \mathrm{Ya}$ Hsin J, Thomasd N, et al. Antihypertensive effects of tannins isolated from traditional Chinese herbs as non-specific inhibitors of angiontensin converting enzyme. Life Sciences- 2003;73:1543-55. 
8. Aguwa CC, Lawal AM. Pharmacologic study on the active principles of Calliandra portoticensis leaf extracts. J Ethnopharmacol. 1994;22(1):63-71.

9. Havsteen B. The biochemistry and medical significance of the flavonoids. Pharmacol Therap. 2002;96:67-202.

10. Hai-Xia L, Shu-Yan H, Xu M, Ke Z, Li W, Zhi-Zhong $M$, et al. The saponin of red ginseng protects the cardiac myocytes against ischemic injury in vitro and in vivo. Phytomed. 2012;1-7(artículo en prensa).

11. Haibo $H$, Jia $X$, Yuanqing $X$, Changcheng $Z$, Hongwu W, Yumin $\mathrm{H}$, et al. Cardioprotective effects of saponins from Panax japonicus on acute myocardial ischemia against oxidative stresstriggered damage and cardiac cell death in rats J Ethnopharmacol. 2012;140:73-82.

12. Shetty K. Biotechnology to harness the benefits of dietary phenolics: focus on Lamiaceae. Asia Pac J Clin Nutr. 1997;6(3):162-71.

13. Katzun G. Farmacologia básica y clínica, octava edición. México: Editorial El Manual Moderno. 2002.

14. Lock O. Investigación fitoquímica; métodos en el estudio de productos naturales. 2da ed. Lima: Fondo Editorial Pontificia Universidad Católica del Perú; 1994

15. Sánchez M, Martinez S, Hernández J, Zuñiga L, Pastelin G, Escalante B. Participación del óxido nítrico y los metabolitos del ácido araquidónico vía citocromo P450 en la regulación de la presión arterial. Arch Cardiol Mex. 2003;73(2):98-104.

16. Ramírez J, Palacios M, Gutiérrez O. Estudio de efecto antihipertensivo de la Salvia scutellarioides en un modelo de ratas hipertensas. Colomb Med. 2006;37(1):53-60.

17. Peotta V, Vasquez E, Meyrelles S. Cardiovascular neural reflexes in L-NAME-induced hypertension in mice. Hypertension. 2001;38:555-9.

18. Howard A, Coffman R, Coffman T, Francois $H$ Makhanova N, Ruiz P, et al. A role for the thromboxane receptor in L-NAME Hypertension. Am J Physiol Renal Physiol. 2008;295:F1096-F1102.
19. Furchgott RF, Vanhoutte PM. Endothelium-derived relaxing and contracting factors. Department of Pharmacology, State University of New York, Brooklyn 11203. 1989

20. Alvarez E, Orallo F. Actividad biológica de los flavonoides (II). Acción cardiovascular y sanguínea. Bioquímica. Documento descargado de http:// www.doymafarma.com el 24/07/2011.

21. Pimentel C, Machado R, Soares R, Luiz C, Lage S. Vasodilator activity of extracts of field Alpinia purpurata (Vieill) K. Schum and A. zerumbet (Pers.) Burtt et Smith cultured in vitro. Braz J Pharm Sci. 2009;45(3).

22. Chen S, Lv G, Zhang X, Liu X, Zhang Z, Zhu Y, Wu $Y$, et al. Anti-hypertensive effects of laiju extract in two different rat models. Asia Pac J Clin Nutr. 2007;16(Suppl 1):309-12.

23. Jiménez E, Hernández F, González M, Tortoriello J, Herrera M. Antihypertensive activity of Salvia elegans Vahl. (Lamiaceae): ACE inhibition and angiotensin II antagonism. J Ethnopharmacol. 2010;130:340-6

24. Adlam D, Bendall J, De Bono J, Alp N, Khoo J, Nicoli T, et al. Relationships between nitric oxide-mediated endotelial function, eNOS coupling and blood pressure revealed by eNOS-GTP cyclohydrolase 1 double transgenic mice. The Physiological Society, The Authors Journal compilation. Oxford 2006.

25. Tenorio F, Del Valle L, Pastelin G. Los flavonoides y el sistema cardiovascular: ¿Pueden ser una alternativa terapéutica?. Departamento de Farmacologia. Instituto Nacional de Cardiología "Ignacio Chávez" (INCICH Juan Badiano Núm. 1, Col. Sección XVI, 14080, Tlalpan, México, D.F.). México 2006

26. Syed A, Samir K, Waqar A, Niaz A. Spasmogenic, spasmolytic and antihypertensive activity of Forsskalea tenacissima L. African J Pharm Pharmacol. University of Malakand. 2010;4(6):381-5,

27. Xu JW, Ikeda K, Yamori Y. Upregulation of endothelial nitric oxide synthase by cianidin-3-glucoside, a typical anthocyanin pigment. Hypertension. 2004;44(2):217-22.
28. Osada K, Funayama M, Fuchi S, Sami M, Ohta Y Kanda $T$, et al. Effects of dietary procyanidins and tea polyphenols on adipose tissue mass and fatty acid metabolism in rats on a high fat diet. $\mathrm{J}$ Oleo Sci. 2006;55(2):79-89.

29. Alarcón-Alonso J, Zamilpaa A, Alarcón F, Herrera M, Tortoriello J, Jimenez-Ferrer E. Pharmacological characterization of the diuretic effect of Hibiscus sabdariffa Linn (Malvaceae) extract. J Ethnopharmacol. 201";139(3):751-6.

30. Yoysungnoen P, Kantasa P, Ingkaninan K. Cardiovascular effects of Tabernae Montana divaricata root extract in anesthetized rats. Thai J Physiol Sci. 2008;21(1).

31. Sukrasno, Sutjiatmo A, Vikasari S, Sari $P$ Anggadiredja K. Antihypertensive effect of Syzygiumpolyanthum(Wight) Walp. leafs extract and combined with Eucheumacottoni and Cycleabarbata Miers. Powder in male Wistar rats using the CODA rat tail-cuff system. School of Pharmacy, Institut Teknologi Bandung, Bandung Indonesia; 2010.

Artículo recibido el 19 de marzo de 2012 y aceptado para publicación el 30 de mayo de 2012.

Agradecimientos:

Al Fondo para la Innovación, la Ciencia y la Tecnologia (FINCYT).

Al Instituto Quimioterápico (IQFARMA).

Conflictos de intereses:

Los autores expresan no tener algún conflicto de interés.

\section{Correspondencia:}

Mg. Lester Dominguez Huarcaya

Facultad de Farmacia y Bioquímica

Correo-e: Idhlester@gmail.com

Celular: 990349345 